\title{
Impact of Age and Baseline NIHSS Scores on Clinical Outcomes in the Mechanical Thrombectomy Using Solitaire FR in Acute Ischemic Stroke Study
}

M.A. Almekhlafi, A. Davalos, A. Bonafe, R. Chapot, J. Gralla, V.M. Pereira, and M. Goyal, on behalf of the STAR Registry Investigators

\section{ABSTRACT}

BACKGROUND AND PURPOSE: Age and stroke severity are inversely correlated with the odds of favorable outcome after ischemic stroke. A previously proposed score for Stroke Prognostication Using Age and NIHSS Stroke Scale (SPAN) indicated that SPAN-100-positive patients (ie, age + NIHSS score $=100$ or more) do not benefit from IV-tPA. If this finding holds true for endovascular therapy, this score can impact patient selection for such interventions. This study investigated whether a score combining age and NIHSS score can improve patients' selection for endovascular stroke therapy.

MATERIALS AND METHODS: The SPAN index was calculated for patients in the prospective Solitaire FR Thrombectomy for Acute Revascularization study: an international single-arm multicenter cohort for anterior circulation stroke treatment by using the Solitaire FR. The proportion with favorable outcome (90-day mRS score $\leq 2)$ was compared between SPAN-100-positive versus-negative patients.

RESULTS: Of the 202 patients enrolled, 196 had baseline NIHSS scores. Fifteen (7.7\%) patients were SPAN-100-positive. There was no difference in the rate of successful reperfusion (Thrombolysis In Cerebral Infarction 2b or 3) between SPAN-100-positive versus -negative groups $(93.3 \%$ versus $82.8 \%$, respectively; $P=.3$ ). Stroke SPAN-100-positive patients had a significantly lower proportion of favorable clinical outcomes (26.7\% versus $60.8 \%$ in SPAN-100-negative, $P=.01$ ). In a multivariable analysis, SPAN-100-positive status was associated with lower odds of favorable outcome (OR, 0.3; 95\% Cl, 0.1-0.9; $P=.04$ ). A higher baseline Alberta Stroke Program Early CT Score and a short onset to revascularization time also predicted favorable outcome in the multivariable analysis.

CONCLUSIONS: A significantly lower proportion of patients with a positive SPAN-100 achieved favorable outcome in this cohort. SPAN-100 was an independent predictor of favorable outcome after adjusting for time to treatment and the extent of preintervention tissue damage according to the Alberta Stroke Program Early CT Score.

ABBREVIATIONS: SPAN = Stroke Prognostication Using Age and NIH Stroke Scale; STAR = Solitaire FR Thrombectomy for Acute Revascularization study

$\mathbf{S}$ troke-related disability remains high at nearly 2 decades since the introduction of IV-tPA as an acute ischemic stroke therapy. ${ }^{1}$ Three recent large randomized trials failed to demonstrate

\section{Received August 28, 2013; accepted after revision November 11.}

From the Departments of Clinical Neurosciences (M.A.A.), and Radiology (M.A.A., M.G.), University of Calgary, Calgary, Alberta, Canada; Faculty of Medicine (M.A.A.) King Abdulaziz University, Jeddah, Saudi Arabia; Department of Neurology (A.D.), University Hospital Germans Trias i Pujol, Badalona, Barcelona, Spain; Department of Neuroradiology (A.B.), Centre Hospitalier Universitaire de Montpellie-Guy de Chauliac, Montpellier, France; Department of Neuroradiology (R.C.), Alfred Krupp Krankenhaus, Essen, Germany; Department of Diagnostic and Interventional Neuroradiology (J.G.), Inselspital, University of Bern, Bern, Switzerland; Department of Neuroradiology (V.M.P.), University Hospital of Geneva, Geneva, Switzerland; and Department for Radiology (M.G.), Hotchkiss Brain Institute, University of Calgary, Calgary, Alberta, Canada.

The STAR study was sponsored by Covidien Neurovascular. Two academic Principal Investigators (J.G., V.M.P.) and an academic Steering Committee supervised the STAR trial design and operations. The Principal Investigators and the Steering Committee interpreted the results and wrote the report. The Principal Investigators and the Steering Committee had full access to the study data and had the final decision to submit for publication. The sponsor of the study was responsible for site management, data management, and safety reporting. Statistical analyses the efficacy of endovascular therapies in improving the 90-day functional outcomes over IV-tPA alone. ${ }^{2-4}$ These trials are criticized for time delays in achieving reperfusion and for the use of dated devices in most patients. Stent retrievers have proved efficacy over the Merci retriever (Concentric Medical, Mountain View, California), ${ }^{5,6}$ but they were used in $<1 \%$ of patients in the recent neutral trials. Therefore, at least 4 multicenter randomized trials of acute stroke endovascular therapy by using stent retrievers are currently recruiting, and other trials are launching soon.

\footnotetext{
were conducted by Jill Schafer, Senior Statistician, and NAMSA, a commercial medical research organization (www.namsa.com). This study is registered with ClinicalTrials.gov (http://www.clinicaltrials.gov), number NCT01327989.

Please address correspondence to Mayank Goyal, MD, FRCPC, Department of Radiology, Seaman Family MR Research Centre, Foothills Medical Centre, 140329th St NW, Calgary, Alberta T2N 2T9, Canada; e-mail: mgoyal@ucalgary.ca

$\equiv$ Indicates article with supplemental on-line table.

Evidence-Based Medicine Level 2.

http://dx.doi.org/10.3174/ajnr.A3855
} 
The importance of patient selection for endovascular therapy of acute ischemic stroke cannot be overemphasized. While controversy exists regarding the optimal imaging technique for patient selection for endovascular therapy, there are proved and readily-available clinical indicators. Among the factors associated with poor functional recovery, age and NIHSS score are most relevant. ${ }^{7}$ This finding led to the derivation of the Stroke Prognostication Using Age and NIHSS Stroke Scale (SPAN) index by adding the patient age in years plus the baseline NIHSS score. Investigators reported that patients in the National Institute of Neurological Disorders and Stroke trial with a score of $\geq 100$ (SPAN-100) did not benefit from IV-tPA therapy, with a higher rate of symptomatic intracranial hemorrhage and poor functional outcome compared with SPAN-100-negative patients. ${ }^{8}$ However, these findings do not take into account the rate of successful recanalization that has been consistently shown to be one of the strongest predictors of favorable stroke outcome. ${ }^{9}$ The National Institute of Neurological Disorders and Stroke trial, ${ }^{10}$ conducted between 1991 and 1994, does not reflect contemporary stroke care; this feature limits its generalizability.

If the SPAN-100 index can identify patients who do not benefit from endovascular therapy, this simple and readily available index will have implications for patient eligibility for these interventions. We assessed the impact of the SPAN-100 index in the large multicenter prospective study for mechanical thrombectomy in acute ischemic stroke, Solitaire FR Thrombectomy for Acute Revascularization (STAR). ${ }^{11}$

\section{MATERIALS AND METHODS}

The STAR registry was a single-arm prospective one conducted in 12 centers in Europe, Canada, and Australia. ${ }^{11}$ Patients were eligible for enrollment if they presented within 8 hours after onset of an acute ischemic stroke due to a proximal intracranial arterial occlusion in the anterior circulation. Key inclusion criteria were the following: age (18 years of age and older and younger than 85 years), NIHSS score of $8-30$, mRS score of $<2$, and a documented occlusion of an intracranial artery on conventional angiography.

\section{Treatment Protocol and Outcomes}

Patients arriving at the hospital within 4.5 hours were treated with IV-tPA in the absence of contraindications, while the rest were referred for primary mechanical thrombectomy. The Solitaire FR (flow restoration) device (Covidien, Irvine, California) was the primary stent retrievers used in the study. Successful revascularization was defined as a TICI score of $2 b$ or more of the target territory with a maximum of 3 passes of the study device per vessel. Rescue therapy was permitted if adequate revascularization (TICI $>2 \mathrm{~b}$ ) had not been achieved.

The primary end point of the STAR registry was the revascularization rate (TICI $2 \mathrm{~b}$ or greater) of the occluded vessel after a maximum of 3 passes of the study device as determined by an independent core laboratory.

\section{SPAN-100 Index}

The SPAN index was calculated by adding age in years to the baseline NIHSS score, as described previously. ${ }^{8}$ Scores were di- chotomized as SPAN-100-positive if the index score was $\geq 100$ and SPAN-100-negative if the index score was $<100$.

\section{Statistical Analysis}

Patients were stratified according to the SPAN-100 index and successful revascularization status. The proportion of patients with favorable clinical outcome (90-day mRS score $\leq 2$ ) was compared between SPAN-100-positive versus -negative. In addition, the rates of symptomatic hemorrhage and procedure-related complications were compared between the 2 groups. A multivariable logistic regression model was fitted for favorable clinical outcome, adjusting for the SPAN-100 index, successful revascularization, baseline ASPECTS, IV-tPA treatment, and time from symptom onset to revascularization. Statistical analysis was performed in SAS, Version 9.3 (SAS Institute, Cary, North Carolina). All tests were 2-tailed with a prespecified $P$ value of .05 .

\section{Role of the Funding Source}

The STAR registry was designed by academic principal investigators and had an academic Steering Committee composed of experts in vascular neurology and interventional neuroradiology. An independent Clinical Events Committee was responsible for the review and validation of all complications. The principal investigators and the Steering Committee interpreted the results and wrote the final report of the study. Study management and funding were provided by Covidien Neurovascular. The STAR registry is registered with ClinicalTrials.gov, number NCT01327989.

The current report is a post hoc analysis that is investigator-driven.

\section{RESULTS}

Of the 202 patients enrolled, 196 had NIHSS scores at baseline. Fifteen patients $(7.7 \%)$ had a SPAN index of $\geq 100$. The On-line Table shows the baseline characteristics of the study groups.

As expected, the SPAN-100-positive group was relatively older, with $60 \%$ being 80 years of age or older. This group also had a higher prevalence of diabetes mellitus, hypertension, atrial fibrillation, and prior history of myocardial infarction compared with SPAN-100-negative patients. There was no difference in the median baseline ASPECTS or the location of arterial occlusions. There were delays (45 minutes on average) in the time from stroke-symptom onset to groin arterial access for the endovascular procedure in the SPAN-100-positive group.

\section{Outcomes}

Successful revascularization was achieved in $88 \%$ of patients in the STAR registry (Table 1). This rate was similar in the SPAN100 -positive versus -negative groups (93.3\% versus $82.8 \%$, respectively; $P=.3$ ). Procedure-related complications were not different between the 2 groups. A significantly lower proportion of patients in the SPAN-100-positive group achieved revascularization within 4.5 hours from stroke-symptom onset compared with the SPAN-100-negative group (20\% versus $47 \%$, respectively; $P=.043$ ). There was no difference in the time interval from CT to TICI $2 \mathrm{~b}$ or 3 or final angiographic run (mean time of 165.7 versus 141.5 minutes in SPAN-100-positive and -negative, respectively; $P=.2$ ).

While there was a suggestion of a higher incidence of any intracranial hemorrhage in the SPAN-100-positive group (27\% ver- 


\begin{tabular}{|c|c|c|c|}
\hline Outcome & $\begin{array}{l}\text { Entire Cohort } \\
\quad(N=202)\end{array}$ & $\begin{array}{c}\text { SPAN-100-Positive } \\
(n=15)\end{array}$ & $\begin{array}{c}\text { SPAN-100-Negative } \\
(n=181)\end{array}$ \\
\hline Median time from stroke onset to groin puncture (min) (range) & $238(72-714)$ & $280(140-450)$ & $235(72-714)$ \\
\hline Successful recanalization (after rescue therapy) as per core lab ${ }^{a}$ & $88 \%(171 / 194)$ & $93 \%(14 / 15)$ & $87 \%(151 / 173)$ \\
\hline Median time from stroke onset to $\mathrm{TICI} 2 \mathrm{~b}$ or 3 (or final DSA run) (min) (range) & $282(100-800)$ & $322(188-483)$ & $282(100-800)$ \\
\hline \multicolumn{4}{|l|}{ Stroke onset to successful revascularization categories } \\
\hline $0-4.5 \mathrm{hr}$ & $46 \%(92 / 201)$ & $20 \%(3 / 15)$ & $47 \%(84 / 180)$ \\
\hline $4.5-8 \mathrm{hr}$ & $50 \%(101 / 201)$ & $73 \%(11 / 15)$ & $49 \%(89 / 180)$ \\
\hline$\geq 8 \mathrm{hr}$ & $4 \%(8 / 201)$ & $7 \%(1 / 15)$ & $4 \%(7 / 180)$ \\
\hline Device- or procedure-related serious adverse events & $7 \%(15 / 202)$ & $7 \%(1 / 15)$ & $8 \%(14 / 181)$ \\
\hline Any intracranial hemorrhage & $19 \%(38 / 202)$ & $27 \%(4 / 15)$ & $19 \%(34 / 181)$ \\
\hline Symptomatic intracranial hemorrhage & $1 \%(3 / 202)$ & $0 \%(0 / 15)$ & $2 \%(3 / 181)$ \\
\hline \multicolumn{4}{|l|}{ 90-Day clinical outcomes } \\
\hline Death from any cause & $7 \%(14 / 202)$ & $27 \%(4 / 15)$ & $6 \%(10 / 181)$ \\
\hline Good functional recovery (modified Rankin Scale score 0-2) & $58 \%(117 / 202)$ & $27 \%(4 / 15)$ & $61 \%(110 / 181)$ \\
\hline
\end{tabular}

${ }^{a}$ Core laboratory missed the primary end point data of 8 subjects.

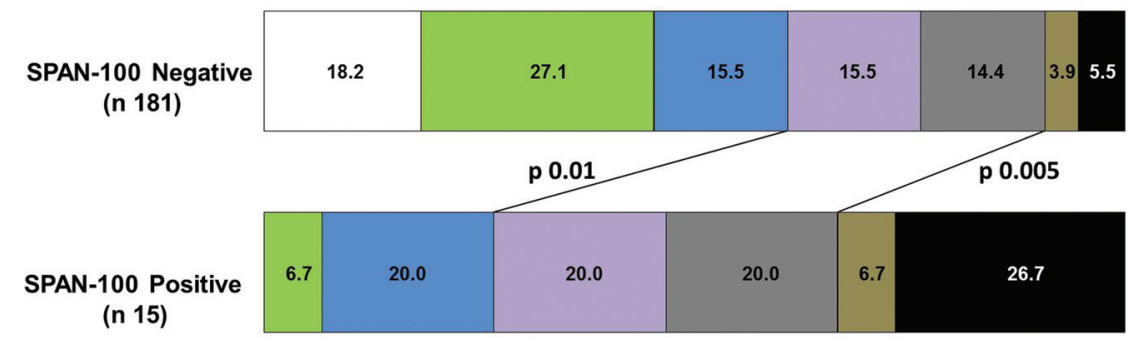

Proportion of patients (\%)

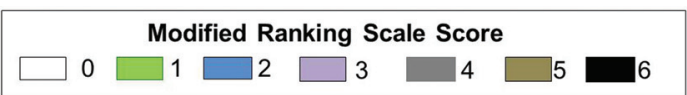

FIG 1. Ninety-day modified Rankin Scale scores. The distribution of 90-day modified Rankin Scale scores among patients according to the SPAN-100 index. The lines indicate the mRS category ( $\mathrm{mRS} 0-2$ and $\mathrm{mRS} 5-6$ ) between SPAN-100 groups. $P$ value refers to the significance level of the $\chi^{2}$ test for proportion comparison.

sus $19 \%$ in the SPAN-100-negative group, $P=.5$ ), none of those patients were symptomatic compared with only $2 \%$ symptomatic intracranial hemorrhages in the SPAN-100-negative group.

None of the SPAN-100-positive patients achieved a 90-day mRS score of zero, and only 1 patient (6.7\%) achieved an mRS score of 1 (Fig $1 ; P=.004$ for the difference in the 90 -day mRS of $\leq 1$ between SPAN-100-positive versus -negative). Similarly, a significantly lower proportion of patients in the SPAN-100-positive group achieved a 90-day mRS score of $\leq 2$ compared with the SPAN-100-negative group (26.7\% versus $60.8 \%$, respectively; $P=.01)$.

In a univariable logistic regression for favorable clinical outcome at 90 days (Table 2), significant predictors were the SPAN100 -positive index $(\mathrm{OR}=0.2 ; 95 \% \mathrm{CI}, 0.1-0.8 ; P=.02)$, baseline ASPECTS $(\mathrm{OR}=1.3 ; 95 \% \mathrm{CI}, 1.1-1.6 ; P=.003)$, and time from stroke onset to successful revascularization $(\mathrm{OR}=0.7 ; 95 \% \mathrm{CI}$, $0.6-0.8 ; P<.001)$. When these predictors were analyzed in a multivariable logistic regression model (Table 2), SPAN-100 remained a significant predictor of favorable outcome (OR 0.3; $95 \%$ CI, 0.1-0.9; $P=.04)$. In addition, the baseline ASPECTS (OR = $1.3 ; 95 \% \mathrm{CI}, 1.04-1.6 ; P=.02)$ and time from stroke onset to successful revascularization $(\mathrm{OR}=0.7 ; 95 \% \mathrm{CI}, 0.6-0.8 ; P<$ $.001)$ were significant. There was no interaction between the
SPAN-100 index and time from stroke onset to successful revascularization.

\section{DISCUSSION}

Patient selection for endovascular acute ischemic stroke therapy remains a challenge. The relatively high rates of successful revascularization are unable to improve the number of patients with favorable clinical outcomes. ${ }^{12}$ This result might be explained, at least in part, by inappropriate patient selection for endovascular interventions. The SPAN100 index is promising to provide a simple and readily available tool to enhance the patient-selection process. In the STAR registry, we found that patients with a SPAN-100-positive index do not benefit from endovascular therapy to the same extent as patients who are SPAN-100-negative. This effect remains significant even after adjusting for those with a limited extent of ischemic change on baseline CT and a short onset to successful revascularization time.

This is the first study to evaluate the SPAN-100 index in a cohort of patients with acute stroke treated with the most recent and effective endovascular devices, stent retrievers. This analysis may have implications for patient selection for future endovascular randomized trials. The findings highlight the importance of selecting appropriate patients for endovascular therapy and achieving successful revascularization quickly to improve the outcome of acute ischemic stroke. Although SPAN-100-positive patients may still achieve favorable outcome if revascularized quickly, the size of the effect in this patient population is expected to be smaller than that in SPAN-100-negative patients. While there was no difference in the baseline ASPECTS for patients in the SPAN-100-positive versus -negative groups, differences in the extent of collateral circulation in those patients might be relevant. The known association between poor collaterals and older age may account for the lack of efficacy of endovascular therapy in this patient population and indicates the importance of collateral assessment in selecting patients for this therapy. Finally, trials for endovascular therapy beyond 4.5 hours from stroke onset may consider ex- 
Table 2: Univariable and multivariable logistic regressions of favorable clinical outcome (mRS $\leq 2)$

\begin{tabular}{|c|c|c|c|c|c|c|}
\hline \multirow[b]{2}{*}{ Variable } & \multicolumn{3}{|c|}{ Univariable } & \multicolumn{3}{|c|}{ Multivariable } \\
\hline & Odds Ratio & $95 \% \mathrm{Cl}$ & $P$ Value & Odds Ratio & $95 \% \mathrm{Cl}$ & $P$ Value \\
\hline SPAN-100-positive & 0.23 & $(0.07-0.77)$ & .02 & 0.25 & $(0.07-0.91)$ & .04 \\
\hline Baseline ASPECTS & 1.32 & $(1.10-1.59)$ & .003 & 1.28 & $(1.04-1.58)$ & .02 \\
\hline Time from stroke onset to $\mathrm{TICI} 2 \mathrm{~b}$ or 3 (or final DSA run, hr) & 0.70 & $(0.58-0.84)$ & $<.001$ & 0.68 & $(0.55-0.84)$ & $<.001$ \\
\hline Primary end point success & 1.81 & $(0.82-3.96)$ & .14 & 1.73 & $(0.74-4.03)$ & .21 \\
\hline IV-tPA administered (vs contraindicated or bridging) & 1.05 & $(0.59-1.87)$ & .87 & 1.27 & $(0.64-2.52)$ & .49 \\
\hline
\end{tabular}

cluding SPAN-100-positive patients on the basis of the results of this analysis.

The significant difference in functional outcome between the SPAN-100 groups has implications for the outcome measures in endovascular stroke trials. While patients in the SPAN-100-positive group were less likely to have $\mathrm{mRS} \leq 2$, the proportion of patients in the intermediate disability categories ( $\mathrm{mRS} 3$ or 4 ) was not different from that in the SPAN-100-negative group. In endovascular trials with no upper age limit for enrollment, patients with a SPAN-100-positive index are likely to be encountered and may dilute any efficacy in the endovascular arm if a dichotomous analysis of outcome is adopted. In these trials, alternative analytic approaches that take into account the entire distribution of the 90-day mRS (eg, shift analysis) ${ }^{13}$ might help capture any treatment effect compared with the traditional dichotomous outcome analysis.

This study has limitations. It was based on the nonrandomized STAR prospective registry. Therefore, the effect of endovascular therapy compared with IV-tPA in SPAN-100-positive versusnegative patients could not be assessed. Approximately $8 \%$ of the STAR registry patients were SPAN-100-positive. This relatively small number may limit the generalizability of the results. While the multivariable logistic regression identified the SPAN-100 index as a predictor of favorable outcome, rapid revascularization of patients with limited early ischemic changes on baseline imaging may still be of benefit, even with SPAN-100-positive status.

\section{CONCLUSIONS}

A significantly lower proportion of patients with a positive SPAN100 index achieved a favorable outcome at 90 days in this cohort. SPAN-100 was an independent predictor of favorable outcome after adjusting for time to treatment and the extent of preintervention tissue damage according to ASPECTS.

\section{ACKNOWLEDGMENTS}

We thank Jill Schafer for her assistance with the statistical analyses.

Disclosures: Antonio Davalos—RELATED: Consulting Fee or Honorarium: Covidien, Comments: moderate, as member of the Steering Committee of the STAR trial, Support for Travel to Meetings for the Study or Other Purposes: Covidien, Comments: moderate, as member of the Steering Committee of the STAR trial, UNRELATED: Consultancy: Lundbeck, Comments: member of the Steering Committee of the Desmoteplase in Acute Ischemic Stroke (DIAS)3 and DIAS4 trials, Grants/ Grants Pending: Covidien, ${ }^{*}$ Comments: The grant is unrestricted and given to a nonprofit private foundation (Fundació Ictus) in order to finance an academic trial, the Randomized Trial of Revascularization with Solitaire FR, Payment for Lectures (including service on Speakers Bureaus): Lundbeck, Comments: member of the Steering Committee of the Desmoteplase in Acute Ischemic Stroke (DIAS)3 and DIAS4 trials. Alain Bonafe-UNRELATED: Consultancy: Covidien. Rene ChapotUNRELATED: Consultancy: Covidien, ${ }^{*}$ MicroVention, ${ }^{*}$ Travel/Accommodations/ Meeting Expenses Unrelated to Activities Listed: Balt,* MicroVention,* Covidien.* Jan Gralla—RELATED: Consulting Fee or Honorarium: Covidien, ${ }^{\star}$ Comments: Global Principal Investigator of the STAR study and consultant. Vitor Mendes PereiraRELATED: Grant: Covidien, ${ }^{*}$ Comments: Principal Investigator of STAR trial, UNRELATED: Consultancy: Covidien.* Mayank Goyal—RELATED: Grant: Covidien/ev3,* Comments: funding for the Endovascular Treatment for Small Core and Anterior Circulation Proximal Occlusion with Emphasis on Minimizing CT to Recanalization Times (ESCAPE) trial;, Consulting Fee or Honorarium: Covidien/ev3, Comments: for teaching engagements and trial design and execution. *Money paid to the institution.

\section{REFERENCES}

1. Mukherjee D, Patil CG. Epidemiology and the global burden of stroke. World Neurosurg 2011;76:S85-90

2. Kidwell CS, Jahan R, Gornbein J, et al. A trial of imaging selection and endovascular treatment for ischemic stroke. $N$ Engl J Med 2013;368:914-23

3. Broderick JP, Palesch YY, Demchuk AM, et al. Endovascular therapy after intravenous t-PA versus t-PA alone for stroke. $N$ Engl J Med 2013;368:893-903

4. Ciccone A, Valvassori L, Nichelatti M, et al. Endovascular treatment for acute ischemic stroke. N Engl J Med 2013;368:904-13

5. Saver JL, Jahan R, Levy EI, et al. Solitaire flow restoration device versus the Merci retriever in patients with acute ischaemic stroke (SWIFT): a randomised, parallel-group, non-inferiority trial. Lancet 2012;380:1241-49

6. Nogueira RG, Lutsep HL, Gupta R, et al. Trevo versus Merci retrievers for thrombectomy revascularisation of large vessel occlusions in acute ischaemic stroke (TREVO 2): a randomised trial. Lancet 2012;380:1231-40

7. Weimar C, Konig IR, Kraywinkel K, et al. Age and National Institutes of Health Stroke Scale score within 6 hours after onset are accurate predictors of outcome after cerebral ischemia: development and external validation of prognostic models. Stroke 2004;35:158-62

8. Saposnik G, Guzik AK, Reeves M, et al. Stroke prognostication using age and NIH Stroke Scale: SPAN-100. Neurology 2013;80:21-28

9. Rha JH, Saver JL. The impact of recanalization on ischemic stroke outcome: a meta-analysis. Stroke 2007;38:967-73

10. Tissue plasminogen activator for acute ischemic stroke: the National Institute of Neurological Disorders and Stroke rt-PA Stroke Study Group. N Engl J Med 1995;333:1581-87

11. Pereira VM, Gralla J, Davalos A, et al. Prospective, multicenter, single-arm study of mechanical thrombectomy using Solitaire flow restoration in acute ischemic stroke. Stroke 2013;44:2802-07

12. Almekhlafi MA, Menon BK, Freiheit EA, et al. A meta-analysis of observational intra-arterial stroke therapy studies using the Merci device, Penumbra system, and retrievable stents. AJNR Am J Neuroradiol 2013;34:140-45

13. Saver JL, Gornbein J. Treatment effects for which shift or binary analyses are advantageous in acute stroke trials. Neurology 2009;72: 1310-15 